\title{
Preparation, Characterization, and Cytotoxicity of Various Chitosan Nanoparticles
}

\author{
Qian Yao, ${ }^{1}$ Wei Liu, ${ }^{1}$ Xiao-Jun Gou, ${ }^{1}$ Xiao-Qiang Guo, ${ }^{1}$ Jun Yan, ${ }^{1}$ Qin Song, \\ Feng-Zheng Chen, ${ }^{1}$ Qi Zhao, ${ }^{1}$ Chao Chen, ${ }^{2}$ and Tian Chen ${ }^{2}$ \\ ${ }^{1}$ Key Laboratory of Sichuan Province in Medicinal Chemistry, Chengdu University, Chengdu 610106, China \\ ${ }^{2}$ Department of Pathogenic Biology, Chengdu Medical College, Chengdu 610500, China \\ Correspondence should be addressed to Tian Chen; cduzyhx@hotmail.com
}

Received 27 June 2013; Revised 25 August 2013; Accepted 26 August 2013

Academic Editor: Xinqing Chen

Copyright (c) 2013 Qian Yao et al. This is an open access article distributed under the Creative Commons Attribution License, which permits unrestricted use, distribution, and reproduction in any medium, provided the original work is properly cited.

Chitosan nanoparticles (CS-NPs) without drug loading have diverse biological activity. In this study, we prepared CS-NPs, CS-NPs solidified by different amount of glutaraldehyde, and CS-NPs modified with either biotin (B-CS-NPs) or biotin and avidin (AB-CS-NPs) and examined their cytotoxicity on HepG2 cells. The morphology and size were measured by transmission electron microscopy and photon correction spectroscopy, respectively. The extent of solidification was validated by the approach of sonication. Biotin connect density on the surface of B-CS-NPs and A-B-CS-NPs was determined by biotin assay kit. The results showed that most of the NPs were round and their mean sizes were all below $300 \mathrm{~nm}$. Biotin connect density of B-CS-NPs and A-BCS-NPs was $2.18 \pm 0.36$ and $1.26 \pm 0.11 \mathrm{~mol}$ biotin $/ \mathrm{mol} \mathrm{CS}$, respectively. At relatively low concentration, CS-NPs with higher extent of solidification exhibited more vigorous inhibitory effect against HepG2 cells than those without solidification. When NPs were incubated with cancer cells for $48 \mathrm{~h}$, compared with CS-NPs, the anticancer activity of B-CS-NPs and A-B-CS-NPs was enhanced significantly $(P<0.05)$. In addition, A-B-CS-NPs showed superior cytotoxicity over B-CS-NPs. This study demonstrates that modification with biotin and avidin may be an efficient way in improving antitumor activity of CS-NPs against hepatic carcinoma.

\section{Introduction}

Chitosan is a biodegradable polymer widely applied in pharmaceutical and medical fields, for example, to fabricate nanoparticles (NPs) and functional chitosan derivatives [14]. Recent studies revealed that chitosan nanoparticles (CSNPs) without drug loading have versatile biological activities. Blank CS-NPs themselves were able to inhibit bacterial proliferation, such as E. coli, S. choleraesuis, S. typhimurium, and $S$. aureus [5]. The possible mechanism is that CS-NPs disrupted the cell membranes of bacteria, leading to the leakage of cytoplasm [6]. Owing to their broad antibacterial spectrum and biocompatibility, blank CS-NPs were applied to the treatment of dentinal tubule infection $[7,8]$. With the assistance of ultrasonic activation, CS-NPs were capable of being delivered to deeper location of dentinal tubule where routine cleaning cannot reach [8]. Apart from antibacterial function, CS-NPs have antifungal activity $[9,10]$, which is much more powerful than chitosan itself $[11,12]$. Besides, CSNPs without any drug incorporation possessed anticancer capability. In vitro cell inhibitory experiments showed that CS-NPs exerted vigorous cytotoxicity toward colon cancer cell line (Calo320), gastric cancer cell line (BGC823), and liver cancer cell line (BEL7402) [13]. CS-NPs exhibited significant antitumor activity against Sarcoma-180 and mouse hepatoma H22 in vivo as well [14]. Surface modified CS-NPs usually obtained some unique properties and showed more potent anticancer effect. For example, N-succinyl-CS-NPs were able to block the proliferation of K562 cells by inducing apoptosis and necrosis of cancer cells $[15,16]$.

Nowadays, biotin modified nanoparticles (NPs) represent an attractive strategy to target tumors characterized by rapid division and aggressive growth [17-20]. Biotin is the ligand of avidin, which is another modifying moiety of NPs to specially combine with cancer cells [21-24]. The interaction between biotin and avidin is the strongest one that has even been 
known [25]. As a result, biotin can serve as a bridge to connect avidin with carriers. And it becomes feasible to construct NPs modified with both biotin and avidin by first linking biotin with NPs, then adding avidin to the binding part of biotins on the surface of NPs. The purpose of designing two ligands modified NPs is that they are expected to possess more potent cytotoxicity, for they are capable of entering cancer cells through different routes mediated by two interactions between receptors existing on tumors and ligands that were bound with NPs. Considering that avidin linked NPs have liver targeted feature $[26,27]$, we chose HepG2 cells, a human hepatic carcinoma cell line, to examine the antitumor activity of blank CS-NPs, biotin modified CS-NPs (B-CS-NPs), and CS-NPs modified by both avidin and biotin (A-B- CS-NPs).

If there is no surface modification, free amino groups outside of CS-NPs will be available to react with aldehyde agents to form solidified NPs, which will not have morphological change upon being exposed to body fluid. Though either modification or solidification is not an indispensable step in the preparation of CS-NPs, including either of the two steps will grant some special features to CS-NPs. The solidifying agents that are most frequently used are formaldehyde and glutaraldehyde. Yet whether the introduction of solidifying process will influence antitumor effect of CS-NPs or not remains unclear. In this study, we also prepared CS-NPs with various solidification degrees and compared their inhibitory activities against HepG2 cells.

\section{Methods}

2.1. Preparation of CS-NPs and Solidified CS-NPs. CS-NPs were prepared according to the method we previously established [28]. Briefly, $5 \mathrm{~mL}$ of $0.2 \%$ (W/V) CS solution was put into a $20 \mathrm{~mL}$ beaker. Under constant stirring, $5 \mathrm{~mL}$ of $20 \%(\mathrm{~W} / \mathrm{V})$ sodium chloride solution was dropped into the beaker using a $1 \mathrm{~mL}$ syringe, with one minute interval after each $1 \mathrm{~mL}$ of solution was added. Then, continuous stirring lasted for about $2 \mathrm{~h}$ until blue opalescence appeared, manifesting the formation of NPs. The NPs suspension was centrifuged at $3,000 \mathrm{~g}$ for $5 \mathrm{~min}$, and the pellet was redispersed in $5 \mathrm{~mL}$ distilled water for future use.

To solidify CS-NPs, before centrifugation, 100, 200, and $300 \mu \mathrm{L}$ of $0.3 \% \mathrm{w} / \mathrm{v}$ glutaraldehyde (GA) solution were dropped into CS-NPs suspension under magnetic agitation, respectively, and the stirring was kept on for $2 \mathrm{~h}$ to ensure the completion of the solidifying reaction. Then, the solution was centrifuged and resuspended as the way described above.

2.2. Validating the Solidification of CS-NPs. To examine the solidifying extent of CS-NPs, turbidity of CS-NPs solutions with different addition of GA was assayed, respectively. Afterward, they were exposed to sonication for $5 \mathrm{~min}$, followed by the second measurement of turbidity. The percentage of turbidity decrease correlates with solidification degree of NPs.

2.3. Preparation of $B-C S-N P s$ and $A-B-C S-N P s$. Biotin modified CS was synthesized conforming to the method we published before [29]. In short, $0.3 \%$ CS solution was obtained by dissolving CS in diluted hydrogen chloride solution, and the $\mathrm{pH}$ was adjusted to 6.5 . Then, $12 \mathrm{mg}$ of sulfosuccinimidobiotin (sulfo-NHS-biotin) was dissolved in $2 \mathrm{~mL}$ of distilled water and dropped to $10 \mathrm{~mL}$ of CS solution under magnetic agitation. The stirring was maintained for $2 \mathrm{~h}$ to guarantee complete biotinylated reaction. Afterwards, the solution was transferred to a dialysis bag with 8,000 dalton molecular cutoff and dialyzed against $1 \mathrm{~L}$ distilled water for $24 \mathrm{~h}$. During the period, water was replaced every $8 \mathrm{~h}$. When small molecular agents were removed by dialysis, the solution was lyophilized and reconstituted in $10 \mathrm{~mL}$ water, from which $5 \mathrm{~mL}$ was withdrawn and fabricated into B-CSNPs in accordance with the method for preparing CS-NPs.

Followed by the acquisition of B-CS-NPs, avidin that was ready to conjugate with half of biotin exposed on the surface was added to the solution to construct A-B-CS-NPs. The added amount was based on the binding ratio of avidin to biotin $1: 4$ [25].

2.4. Determination of Biotin Content on the Surface. Biotin content on the surface of B-CS-NPs and A-B-CS-NPs was determined in accordance with manual brochure of biotin assay kit (Pierce Biotechnology, Inc). Biotin connect density was calculated in the light of the formula we previously reported [29].

2.5. Measurement of Morphology and Size. The prepared NPs, including CS-NPs, B-CS-NPs, and A-B-CS-NPs, were stained with $2 \%$ phosphotungstic acid, dried on copper grids overnight, and examined under a transmission electron microscopy (TEM; JTM-1230, Japan), respectively. The size of all batches was measured using a photon correction spectroscopy (PCS; Malvern Instruments, UK). Each sample was assayed in triplicate.

2.6. Cytotoxicity against HepG2 Cells. HepG2 cells were cultured in RPMI-1640 medium supplemented with $15 \%$ fetal bovine serum (FBS) and grew in a water jacket $\mathrm{CO}_{2}$ incubator (Thermo Forma, USA) with an afflux of $5 \% \mathrm{CO}_{2}$ at $37^{\circ} \mathrm{C}$.

When the cellular confluence reached $80 \%$, cells were washed by $3 \mathrm{~mL}$ of PBS and harvested with 1\% trypsin solution for $1 \mathrm{~min}$. Then, culture medium containing 15\% FBS was added to terminate trypsin function. After cell density was adjusted to $1 \times 10^{5}$ cells $/ \mathrm{mL}$, cells were seeded onto 96 well plate, one well loaded with $100 \mu \mathrm{L}$ cellular suspension, and were fostered in incubator for $24 \mathrm{~h}$. Subsequently, fresh complete culture medium replaced the old one. And 2, 4, and $6 \mu \mathrm{L}$ of CS-NPs solidified by different amounts of GA, B-CSNPs, and A-B-CS-NPs, corresponding to the final concentration of 18,36 , and $54 \mathrm{mg} / \mathrm{mL}$ in well, were added to the plate, respectively. The NPs were incubated with cancer cells for 48 and $72 \mathrm{~h}$, respectively. Each level of formulas was repeated on 5 wells. Culture medium, as control group, was manipulated in the same manner. Afterwards, cell viability was assayed using 3-(4,5-dimethylthiazol-2-yl)-2,5-diphenyltetrazolium bromide (MTT) method. The inhibitory rates were calculated by comparing the viability with control group. 


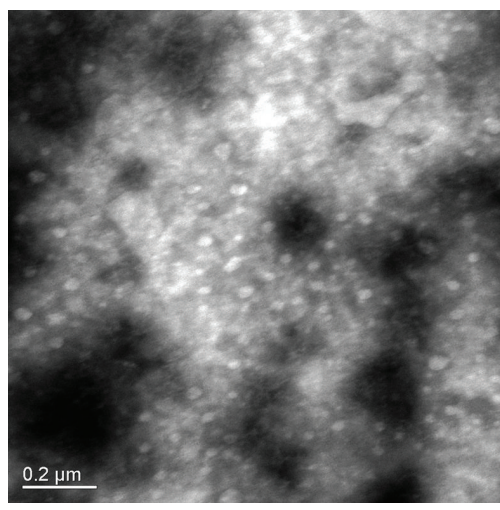

(a)

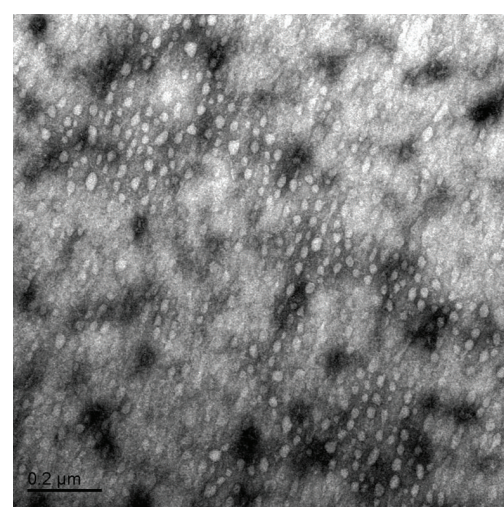

(b)

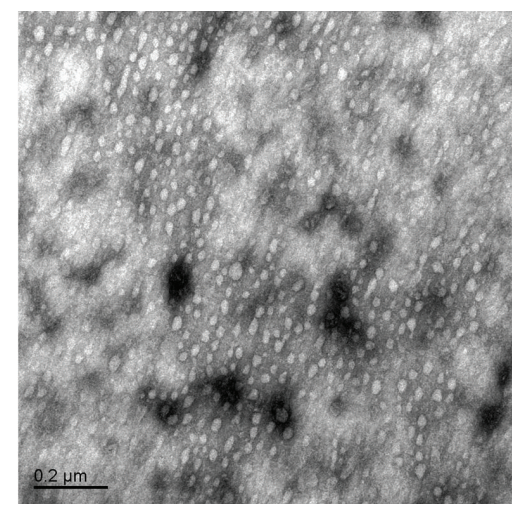

(c)

FIgURE 1: TEM photos of (a) CS-NPs (b) B-CS-NPs, and (c) A-B-CS-NPs.

TABLE 1: Turbidity of CS-NPs with different addition of GA before and after sonication (mean $\pm \mathrm{SD}, n=3$ ).

\begin{tabular}{lccc}
\hline $\begin{array}{l}\text { Glutaraldehyde } \\
\text { added }(\mu \mathrm{L})\end{array}$ & $\begin{array}{c}\text { Before } \\
\text { sonication }\end{array}$ & $\begin{array}{c}\text { After } \\
\text { sonication }\end{array}$ & $\begin{array}{l}\text { Turbidity } \\
\text { decrease } \%\end{array}$ \\
\hline 0 & $0.372 \pm 0.007$ & 0.000 & 100.00 \\
100 & $0.378 \pm 0.006$ & $0.309 \pm 0.005$ & $18.25 \pm 0.46$ \\
200 & $0.371 \pm 0.009$ & $0.350 \pm 0.007$ & $5.66 \pm 0.44$ \\
300 & $0.374 \pm 0.003$ & $0.363 \pm 0.003$ & $2.94 \pm 0.28$ \\
\hline
\end{tabular}

\section{Results and Discussion}

3.1. Validating the Solidification of CS-NPs. Solidifying is not a must for the preparation of CS-NPs, for CS is insoluble in the medium of $\mathrm{pH}$ above 7.0. What NPs benefit from solidification is that they are able to maintain original morphology when delivered to blood circulation. In this study, solidifying extent of CS-NPs was examined by the approach of sonication. The turbidity of CS-NPs with the addition of different amount of GA before and after sonication was displayed in Table 1. It indicated that before sonication, the turbidity of CS-NPs at various extents of solidification was similar to each other, while after sonication, the turbidity of CS-NPs without GA declined to zero, implying that they were totally destroyed by sonication and dissolved in water. With the increase of added GA, the decrease of turbidity caused by sonication became negligible, manifesting that since more CS-NPs were solidified, sonication imposed less effect on CS-NPs. In general, a conclusion can be drawn that the more GA was added, the higher extent of solidification would be achieved.

\subsection{Determination of Biotin Content. Biotin connect density} on the surface of B-CS-NPs and A-B-CS-NPs was $2.18 \pm 0.36$ and $1.26 \pm 0.11 \mathrm{~mol}$ biotin/mol CS, respectively, showing that approximately $40 \%$ of biotins exposed outside of B-CS-NPs had linked with avidins to form A-B-CS-NPs.

3.3. Morphology and Size. The TEM photos of CS-NPs, BCS-NPs, and A-B-CS-NPs (Figure 1) illustrated that most NPs were round with reasonable uniformity. The size measured by PCS of unsolidified CS-NPs and those solidified by 100 , 200 , and $300 \mu \mathrm{L}$ of GA solution was $257,286,282$, and $291 \mathrm{~nm}$, respectively. The uniformity evidenced by polydispersity was $0.225,0.215,0.207$, and 0.212 , respectively. It implied that due to longer stirring to facilitate solidifying reaction, introduction of GA slightly elevated the size of NPs. Nevertheless, the amount of GA had no impact on diameter. The mean size of B-CS-NPs and A-B-CS-NPs was 238 and 286, respectively, and the correlated polydispersity was 0.124 and 0.148 , showing higher uniformity than CS-NPs. Biotin decoration of CS reduced the viscosity of CS, thereby it may contribute to the production of more homogeneous particles. The diameters assayed by PCS were much larger than apparent sizes displayed in photos, owing to the drying process during taking morphological photos that shrank the NPs dramatically.

3.4. Cytotoxicity against HepG2 Cells. The inhibitory rates of unsolidified CS-NPs and those solidified by 100, 200, and $300 \mu \mathrm{L}$ GA solution after being incubated with HepG2 cells for 48 and $72 \mathrm{~h}$ were shown in Figure 2 . It can be seen that the inhibitory activity of NPs at $72 \mathrm{~h}$ was generally lower than at $48 \mathrm{~h}$, implying that cancer cells NPs failed to kill at $48 \mathrm{~h}$ continuously proliferated. In addition, the inhibitory rate elevated with the increase of NPs concentration in culture medium. CS-NPs at low dose, such as $18 \mu \mathrm{g} / \mathrm{mL}$, lost their anticancer ability after acting on the cells for $72 \mathrm{~h}$ (Figure 2). In most cases, CS-NPs at various extents of solidification were similar in terms of inhibitory activity. However, when NPs were cocultured with the cancer cells at $18 \mu \mathrm{g} / \mathrm{mL}$ for $48 \mathrm{~h}$, the cytotoxicity of those solidified by 200 and $300 \mu \mathrm{L}$ of GA was significantly higher than CS-NPs without solidification $(P<0.05)$. The same consequence occurred as NPs were incubated with HepG2 cells at $36 \mu \mathrm{g} / \mathrm{mL}$ for $72 \mathrm{~h}$. It seemed that at relatively low concentration, CS-NPs with higher extent of solidification possessed more vigorous antitumor activity than unsolidified CS-NPs. NPs without solidification at low level were degraded by enzymes secreted from HepG2 cells, which could be considered a contributory factor to the declined cytotoxicity.

The cytotoxicity of CS-NPs, B-CS-NPs, and A-B-CSNPs cocultured with HepG2 cells for 48 and $72 \mathrm{~h}$ was 


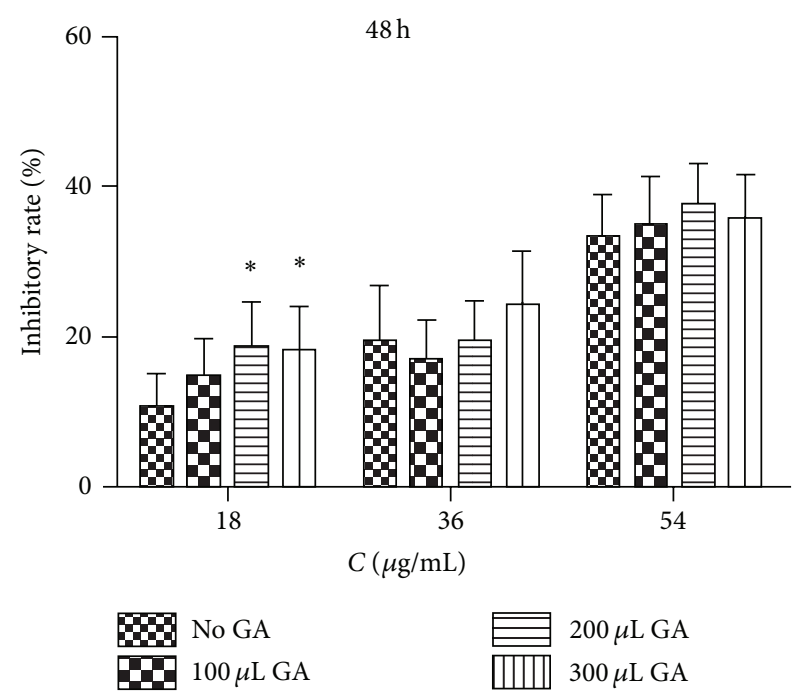

(a)

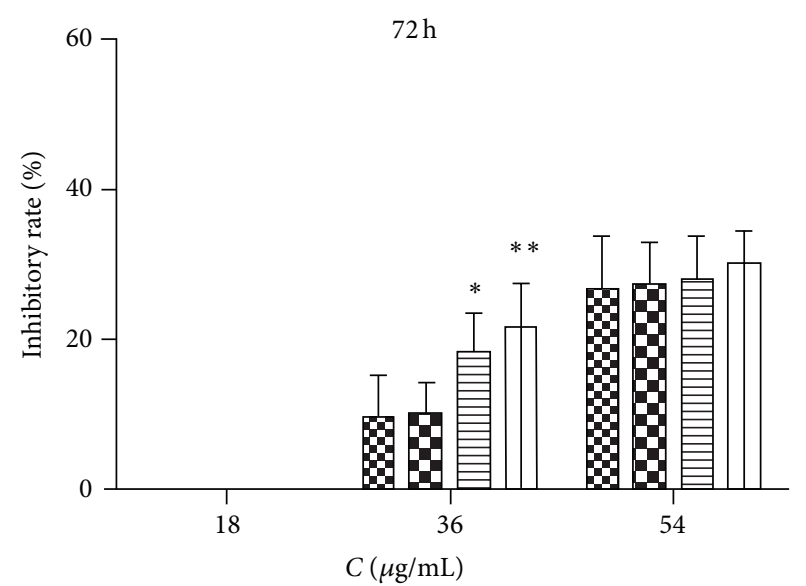

Q07 No GA
$200 \mu \mathrm{L} \mathrm{GA}$

[IIII $300 \mu \mathrm{L} \mathrm{GA}$

(b)

Figure 2: Inhibitory rates against HepG2 cells of CS-NPs without solidification and those solidified by 100, 200, and 300 $\mu$ L GA, after being incubated with the cells for (a) $48 \mathrm{~h}$ and (b) $72 \mathrm{~h}$. The data represent mean $\pm \mathrm{SD}(n=5) .{ }^{*} P \leq 0.05,{ }^{* *} P \leq 0.01$, compared to unsolidified CS-NPs.

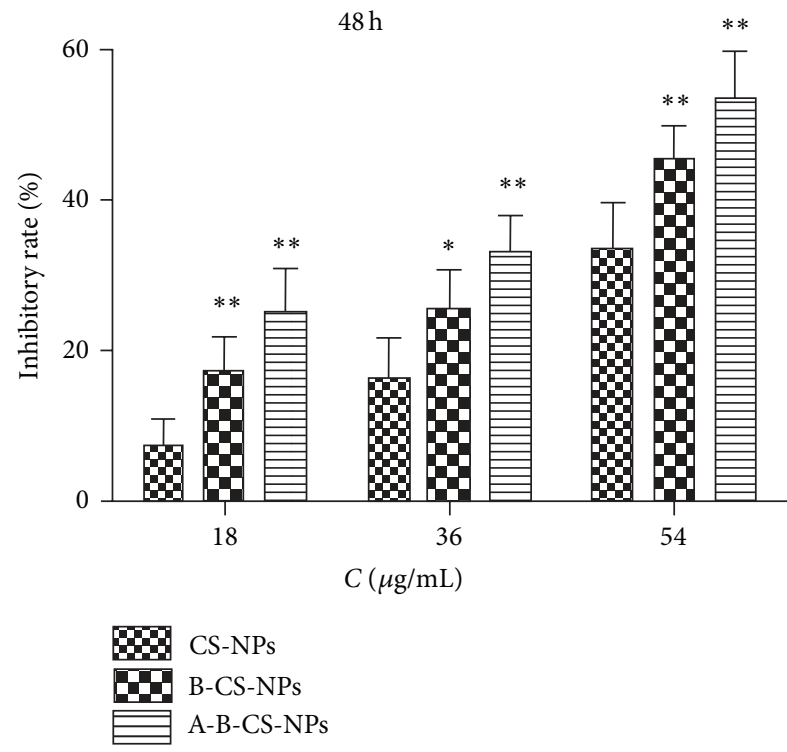

(a)
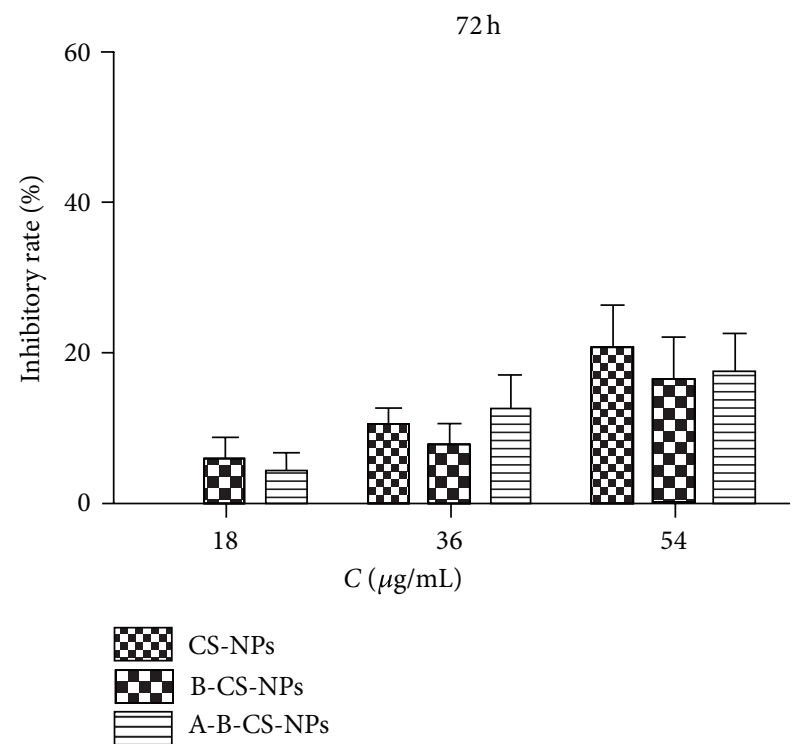

(b)

Figure 3: Inhibitory rates against HepG2 cells of CS-NPs, B-CS-NPs, and A-B-CS-NPs after being incubated with cancer cells for (a) $48 \mathrm{~h}$ and (b) $72 \mathrm{~h}$. The data represent mean $\pm \mathrm{SD}(n=5) .{ }^{*} P \leq 0.05,{ }^{* *} P \leq 0.01$, compared with CS-NPs.

displayed in Figure 3. Similar to the above, the inhibitory activity of NPs sharply decreased after they combated with cancer cells for $72 \mathrm{~h}$. And there was no obvious difference of cytotoxicity among the three NPs at that time. However, when the NPs were incubated with cells for $48 \mathrm{~h}$, B-CSNPs and A-B-CS-NPs substantially blocked more cancer cell growth than CS-NPs at any level $(P<0.05)$, demonstrating that CS-NPs modified by either biotin or biotin and avidin significantly enhanced their antitumor activity. Furthermore, A-B-CS-NPs were more effective in slowing down cancer cell proliferation than B-CS-NPs. As A-B-CS-NPs were modified with two ligands, it may provide the possibility that this kind of NPs entered cells through two different routes, causing more drugs to fight against cancer cells and displaying more vigorous cytotoxicity.

\section{Conclusion}

CS-NPs solidified by different amounts of GA and CS-NPs modified with either biotin (B-CS-NPs) or biotin and avidin 
(A-B-CS-NPs) were prepared in this study. It demonstrated that more GA involved brought about higher solidifying degree of CS-NPs. Biotin connect density of B-CS-NPs and A-B-CS-NPs was $2.18 \pm 0.36$ and $1.26 \pm 0.11 \mathrm{~mol}$ biotin $/ \mathrm{mol}$ CS, respectively. TEM photos declared that most of NPs were round and their mean sizes measured by PCS were all below $300 \mathrm{~nm}$. Cytotoxicity experiment exclaimed that at relatively low concentration, CS-NPs with higher extent of solidification exhibited more potent inhibitory activity against HepG2 cells than unsolidified CS-NPs. When cocultured with cancer cells for $48 \mathrm{~h}$, compared with CS-NPs, B-CS-NPs and A-BCS-NPs significantly suppressed more cancer cell growth. And the anticancer activity of A-B-CS-NPs seemed more potent than that of B-CS-NPs. This study demonstrates that A-B-CS-NPs may be exploited as either an excellent candidate or a potential vehicle for the treatment of hepatic carcinoma. Further experiments need to be conducted to clarify how various CS-NPs act on other cancer cell lines and normal cell lines and the feasibility to be applied in clinical.

\section{Acknowledgments}

The authors thank Professor Zheng-Hua Chen and Professor Jiu-Jin $\mathrm{Xu}$ at Genetics and Developmental Biology, Chinese Academy of Sciences, for their technical assistance. This work was financially supported by the National Natural Science Foundation of China (no. 81173637) and the Key Disciplines Grants Program of Chengdu Medical College (no. CYXK2012005).

\section{References}

[1] T. F. Jiao, J. Zhou, J. Zhou, G. LiHua, Y. Y. Xing, and X. Li, "Synthesis and characterization of chitosan-based schiff base compounds with aromatic substituent groups," Iranian Polymer Journal, vol. 20, no. 2, pp. 123-136, 2011.

[2] A. G. Luque-Alcaraz, J. Lizardi, F. M. Goycoolea et al., "Characterization and antiproliferative activity of nobiletin-loaded chitosan nanoparticles," Journal of Nanomaterials, vol. 2012, Article ID 265161, 7 pages, 2012.

[3] H. Katas, N. N. S. N. Dzulkefli, and S. Sahudin, "Synthesis of a new potential conjugated TAT-peptide-chitosan nanoparticles carrier via disulphide linkage," Journal of Nanomaterials, vol. 2012, Article ID 134607, 7 pages, 2012.

[4] P. R. Chen and Y. J. Chuang, "The development of conductive nanoporous chitosan polymer membrane for selective transport of charged molecules," Journal of Nanomaterials, vol. 2013, Article ID 980857, 6 pages, 2013.

[5] L. Qi, Z. Xu, X. Jiang, C. Hu, and X. Zou, "Preparation and antibacterial activity of chitosan nanoparticles," Carbohydrate Research, vol. 339, no. 16, pp. 2693-2700, 2004.

[6] A. Shrestha, S. Zhilong, N. K. Gee, and A. Kishen, "Nanoparticulates for antibiofilm treatment and effect of aging on its antibacterial activity," Journal of Endodontics, vol. 36, no. 6, pp. 1030-1035, 2010.

[7] A. Shrestha and A. Kishen, "The effect of tissue inhibitors on the antibacterial activity of chitosan nanoparticles and photodynamic therapy," Journal of Endodontics, vol. 38, no. 9, pp. 1275-1278, 2012.
[8] A. Shrestha, S.-W. Fong, B.-C. Khoo, and A. Kishen, "Delivery of antibacterial nanoparticles into dentinal tubules using highintensity focused ultrasound," Journal of Endodontics, vol. 35, no. 7, pp. 1028-1033, 2009.

[9] L. Y. Ing, N. M. Zin, A. Sarwar, and H. Katas, "Antifungal activity of chitosan nanoparticles and correlation with their physical properties,' International Journal of Biomaterials, vol. 2012, Article ID 62398, 9 pages, 2012.

[10] M. G. Guerra-Sánchez, J. Vega-Pérez, M. G. Velázquez-del Valle, and A. N. Hernández-Lauzardo, "Antifungal activity and release of compounds on Rhizopus stolonifer (Ehrenb.:Fr.) Vuill. by effect of chitosan with different molecular weights," Pesticide Biochemistry and Physiology, vol. 93, no. 1, pp. 18-22, 2009.

[11] Z. Zhong, R. Chen, R. Xing et al., "Synthesis and antifungal properties of sulfanilamide derivatives of chitosan," Carbohydrate Research, vol. 342, no. 16, pp. 2390-2395, 2007.

[12] K. Ziani, I. Fernández-Pan, M. Royo, and J. I. Maté, "Antifungal activity of films and solutions based on chitosan against typical seed fungi," Food Hydrocolloids, vol. 23, no. 8, pp. 2309-2314, 2009.

[13] L. Qi, Z. Xu, X. Jiang, Y. Li, and M. Wang, "Cytotoxic activities of chitosan nanoparticles and copper-loaded nanoparticles," Bioorganic \& Medicinal Chemistry Letters, vol. 15, no. 5, pp. 1397-1399, 2005.

[14] L. Qi and Z. Xu, "In vivo antitumor activity of chitosan nanoparticles," Bioorganic \& Medicinal Chemistry Letters, vol. 16, no. 16, pp. 4243-4245, 2006.

[15] H. Luo, H. Su, X. Wang, L. Wang, and J. Li, "N-Succinylchitosan nanoparticles induced mitochondria-dependent apoptosis in K562," Molecular and Cellular Probes, vol. 26, no. 4, pp. 164-169, 2012.

[16] H. Luo, J. Li, and X. Chen, "Antitumor effect of N-succinylchitosan nanoparticles on K562 cells," Biomedicine \& Pharmacotherapy, vol. 64, no. 8, pp. 521-526, 2010.

[17] M. Li, J. W. Y. Lam, F. Mahtab et al., "Biotin-decorated fluorescent silica nanoparticles with aggregation-induced emission characteristics: fabrication, cytotoxicity and biological applications," Journal of Materials Chemistry B, vol. 1, no. 5, pp. 676684, 2013.

[18] M. Patel, R. K. Vadlapatla, S. Shah, and A. K. Mitra, "Molecular expression and functional activity of sodium dependent multivitamin transporter in human prostate cancer cells," International Journal of Pharmaceutics, vol. 436, no. 1-2, pp. 324-331, 2012.

[19] G. Russell-Jones, K. McTavish, J. McEwan, J. Rice, and D. Nowotnik, "Vitamin-mediated targeting as a potential mechanism to increase drug uptake by tumours," Journal of Inorganic Biochemistry, vol. 98, no. 10, pp. 1625-1633, 2004.

[20] J. Chen, S. Chen, X. Zhao, L. V. Kuznetsova, S. S. Wong, and I. Ojima, "Functionalized single-walled carbon nanotubes as rationally designed vehicles for tumor-targeted drug delivery," Journal of the American Chemical Society, vol. 130, no. 49, pp. 16778-16785, 2008.

[21] M. Chinol, O. De Cobelli, G. Trifirò et al., "Localization of avidin in superficial bladder cancer: a potentially new approach for radionuclide therapy," European Urology, vol. 44, no. 5, pp. 556-559, 2003.

[22] Y. Hama, Y. Urano, Y. Koyama, P. L. Choyke, and H. Kobayashi, "Targeted optical imaging of cancer cells using lectin-binding BODIPY conjugated avidin," Biochemical and 
Biophysical Research Communications, vol. 348, no. 3, pp. 807813, 2006.

[23] Y. Hama, Y. Urano, Y. Koyama et al., "A target cell-specific activatable fluorescence probe for in vivo molecular imaging of cancer based on a self-quenched avidin-rhodamine conjugate," Cancer Research, vol. 67, no. 6, pp. 2791-2799, 2007.

[24] M. Ogawa, N. Kosaka, M. R. Longmire, Y. Urano, P. L. Choyke, and H. Kobayashi, "Fluorophore-quencher based activatable targeted optical probes for detecting in vivo cancer metastases," Molecular Pharmaceutics, vol. 6, no. 2, pp. 386-395, 2009.

[25] M. S. Celej, G. G. Montich, and G. D. Fidelio, "Conformational flexibility of avidin: the influence of biotin binding," Biochemical and Biophysical Research Communications, vol. 325, no. 3, pp. 922-927, 2004.

[26] S. F. Rosebrough, "Pharmacokinetics and biodistribution of radiolabeled avidin, streptavidin and biotin," Nuclear Medicine and Biology, vol. 20, no. 5, pp. 663-668, 1993.

[27] B. Schechter, R. Silberman, R. Arnon, and M. Wilchek, “Tissue distribution of avidin and streptavidin injected to mice. Effect of avidin carbohydrate, streptavidin truncated and exogenous biotin," European Journal of Biochemistry, vol. 189, no. 2, pp. 327331, 1990.

[28] Q. Yao, L. Gan, S. Hou et al., "Development and biodistribution of trans-resveratrol loaded chitosan nanoparticles with free amino groups," Latin American Journal of Pharmacy, vol. 31, no. 7, pp. 1038-1042, 2012.

[29] Q. Yao, S.-X. Hou, X. Zhang, G. Zhao, X.-J. Gou, and J.-Z. You, "Preparation and characterization of biotinylated chitosan nanoparticles," Acta Pharmaceutica Sinica, vol. 42, no. 5, pp. 557-561, 2007. 

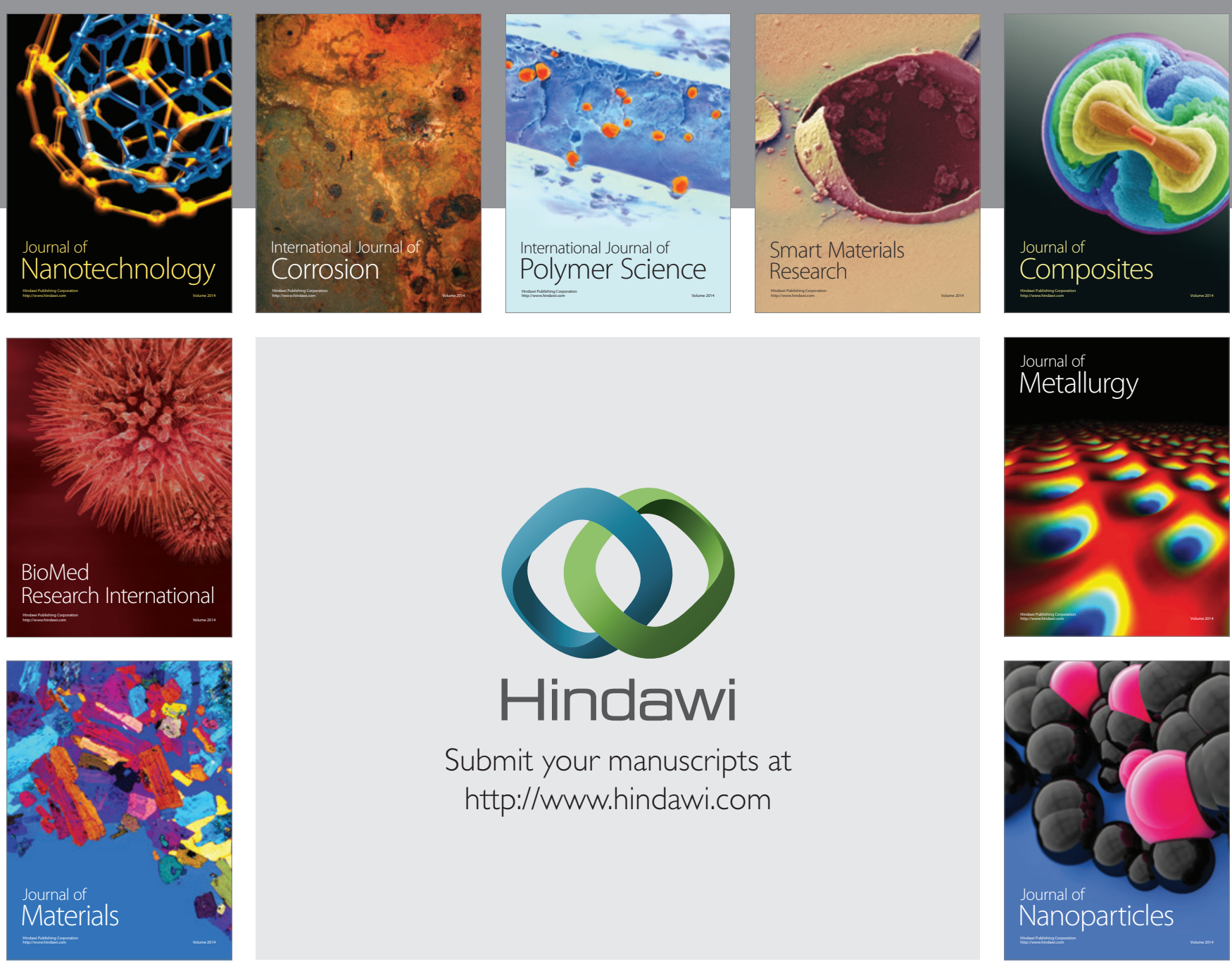

Submit your manuscripts at http://www.hindawi.com
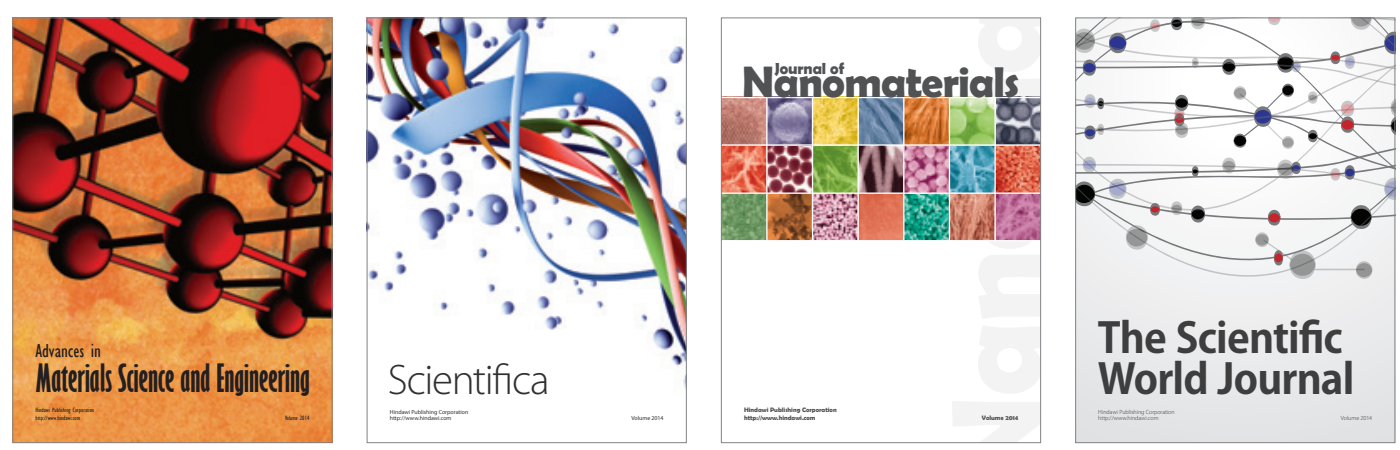

\section{The Scientific World Journal}
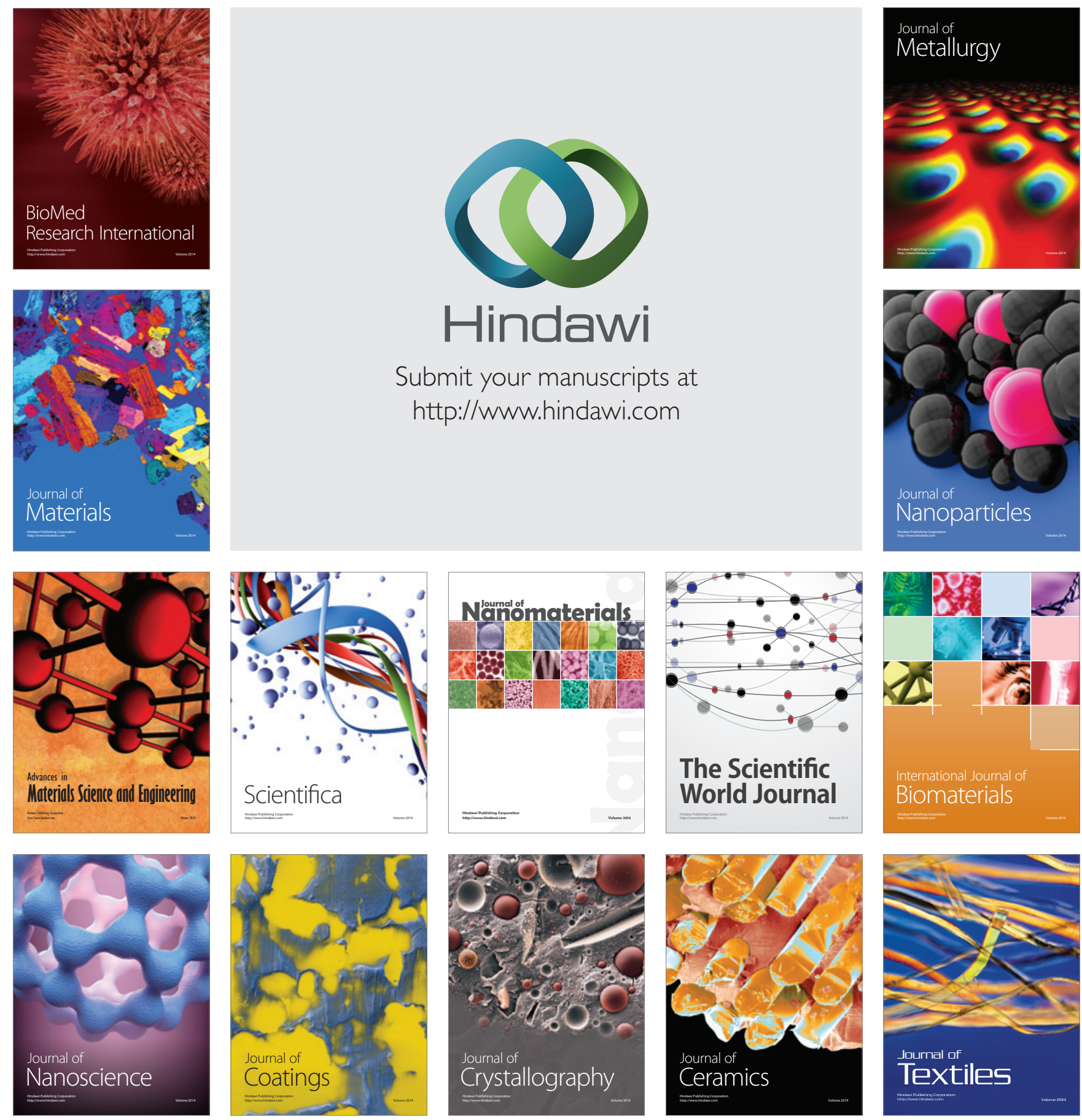